\title{
MJN EFFECTIVENESS OF A NEED BASED ORAL CARE PROTOCOL ON ORAL HEALTH STATUS, OCCURRENCE AND PROGRESSION OF PNEUMONIA AMONG VENTILATED PATIENTS
}

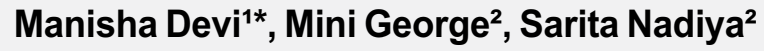 \\ ${ }^{1}$ Maharishi Markandesa College of Nursing, Maharishi Markandeshwar Deemed to University Mullana, \\ Ambala, Haryana, India \\ ${ }^{2}$ College of Nursing, Institute of liver and biliary sciences, Vasankunj, New Delhi, India \\ *Corresponding Author's Email: manisha94saklani@gmail.com
}

\begin{abstract}
Introduction: Ventilated patients with chronic liver diseases are at more risk of developing gingival bleeding and oral mucosal lesion. Purposes: The objective of the present study is to evaluate the Effectiveness of a Need Based Oral Care Protocol on Oral Health Status, Occurrence and Progression of Pneumonia among ventilated patients. Methods: Quasi experimental time series design was used. Fifty ventilated patients were randomly allocated to study $(n=27)$ and comparison $(n=23)$ groups. Severity of illness was assessed by APACHE II score. Occurrence of Pneumonia was assessed using Microbiological profile. The CPIS score was assessed for assessing Progression of Pneumonia and to rule out HAP. Need for oral care and Oral Health Status was assessed daily in terms of frequency of oral care as assessed by MBOAS score. Need based Oral care as per American Association of Critical Care Nurse was provided by the researcher to the patients in the study group. Comparison group received routine oral care as per the existing guidelines by the staff on duty. Assessment of the oral health status was done using MBOAS. Results: The findings related to Oral Health Status showed that the mean MBOAS scores differed significantly $(F=5.82, P=0.001)$, across the time; and across the groups over time $(F=19.16, p=0.001)$. The results of Microbiological Profile revealed that the most common causative organism for VAP in both the Study and Comparison group on day three as well as on day six were Klebsiella pneumoniae, Staphylococcus, Acinetobacter baumannii, Pseudomonas and gram-negative bacteria. In terms of occurrence of Pneumonia there was statistically significant difference found between the groups on day three $\left(\chi^{2}=5.83, p=0.05\right)$ and on day six $\left(\chi^{2}=7.40, p=0.02\right)$. There was significant difference seen between the groups in the Progression of Pneumonia with time from day 1 to $6(F=39.90 ; p=0.001)$, and also across the groups over time $(F=5.14, p=0.03)$. A significant relationship $(r=0.66, p=0.001)$ seen between Severity of Illness with Progression of Pneumonia on day six in the Study group. Conclusion: The findings indicate that the Need Based Oral Care Protocol was effective in improving the Oral Health Status and reducing the occurrence and Progression of Pneumonia among ventilated patients with CLD.
\end{abstract}

Keywords: Oral Health Status; Occurrence of Pneumonia; Progression of Pneumonia; Ventilated Patients; Need Based Oral Care

\section{INTRODUCTION}

Critical care is a term used to describe the care of critically ill patients whose clinical condition is unstable or potentially unstable (Marshall et al., 2017). Critically ill patients frequently require mechanical ventilation because of their diseases condition like severe respiratory failure and hemodynamic instability. Most patients require mechanical ventilation for some time, but some may require for a longer duration. Despite the fact that while a ventilator is often a life saving measure, it can frequently cause complications, including the nosocomial infection like Ventilator associated pneumonia (Koenig \& Truwit, 2006).

In mechanically ventilated patients the risk of developing Ventilator Associated Pneumonia is increased to $6-20 \%$ (Joseph et al., 2009). VAP is the 
most common and lethal infection in patient requiring mechanical ventilation. Ventilator-associated pneumonia is defined as pneumonia that develops while a patient is receiving mechanical ventilation, usually positivepressure ventilation delivered via an endotracheal tube for support during acute respiratory failure (Antonelli et al., 2011). It refers to the Pneumonia that occurs in patients who have been on mechanical ventilation for more than 48 hours. When Pneumonia develops in patients within 48 hours of intubation it is then known as Hospital Acquired Pneumonia (Davis, 2006). The most frequent causative organisms of VAP are Pseudomonas (5.68\%), Gram- negative bacteria (4.93\%), Klebsiella pneumonia (2.89\%), Staphylococcus aureus susceptible to Methicillin $(2.86 \%)$, Candidiasis $(2.57 \%)$, Streptococcus pneumoniae (2.32\%), Escherichia coli (1.83\%), Haemophilus influenzae (1.74\%), and Staphylococcus aureus resistant to Methicillin (1.16\%) (Diez et al., 2017).

A liver disease is a serious issue and approximately $15 \%$ of patients with liver diseases require admission to ICU. The patients with chronic liver diseases may land up with serious complication like sepsis, variceal bleeding, hepatic encephalopathy, spontaneous bacterial peritonitis and hepato-renal syndrome. The patients with liver diseases admitted in ICU require respiratory support. Patients with Liver Disease who require mechanical ventilation are high risk patients and having poor survival with a mortality rate of $89 \%$. Attention to oral health in critically ill patients in the ICU is essential especially the patients with Chronic Liver Diseases. The oral microbial flora of mechanically ventilated patients gradually changes especially the patients with Chronic Liver Diseases. The oral cavity of patients with Chronic Liver disease reflect the changes in Liver dysfunction in the form of mucosal membrane jaundice, bleeding disorders, petechiae, increased vulnerability to bruising, gingivitis, gingival bleeding even in response to minimal handling (Kannan et al., 2018). Panov \& Krasteva, (2011) stated that patients with Chronic Liver diseases results in mucosal lesion and gingival bleeding so handling of oral mucosa should be done cautiously.

\section{METHODOLOGY}

\section{Research Design}

Quasi experimental time series design was used to evaluate the effectiveness of Need based oral care protocol in the month of April 2020 in the state of
Haryana, India.

\section{Research Setting}

The study was conducted at Institute of Liver and Biliary Sciences New Delhi.

\section{Population}

Ventilated Patients with Endotracheal Intubation for Ventilations admitted in Liver Coma Intensive Care Unit of Institute of Liver and Biliary Sciences.

\section{Sampling Technique}

Random allocation was used to assign the subjects into Study and Comparison group by using a computergenerated table.

\section{Sample Size}

Sample size for the present study was estimated before starting of the study. Similar research studies were reviewed, and sample size was estimated from the study, where it was seen that there was $75 \%$ improvement in the Experimental Group and 60\% in the Comparison Group. With an alpha of $5 \%$ and power of $90 \%$ and assuming that the baseline values of Oral Care Assessment in Ventilated Patients are same or comparable, the sample size was calculated with reference to Moustafa et al., (2016). The sample size calculation showed that 32 patients were needed to be randomly allocated as 16 in each group. Further, assuming a $20 \%$ dropout or mortality rate, it was decided to enroll 20 patient each in Study and Comparison group of this study.

\section{Data collection Tools and Techniques}

In the present study followings tools are used to collect the data.

\section{APACHE II Score}

APACHE II score was developed by Knaus and others in 1985. APACHE II score was a scale modified from the earlier version of the APACHE score and used to assess the Severity of Illness in patients within 24 hours of admission of patient to an Intensive Care Unit. The parameters assessed in APACHE II includes Temperature, Mean arterial pressure, Heart rate, Respiratory rate, Partial pressure of oxygen, Arterial $\mathrm{pH}$, Serum Sodium, Serum Potassium, Serum Creatinine, Hematocrit, WBC count, Glasgow coma score and Age. Each variable is weighted from 0-4 with 
higher Score denoting an increasing deviation from normal. The maximum Score is 71 and minimum score is 0 . The scale is used to predict the mortality from the Severity of Illness. The Inter-Rater Reliability of the APACHE II score was established by the author at $(r=0.08 p=0.005)$. In the present study APACHE II score was used when patient enrolled in the study to assess the severity of illness.

\section{Clinical Pulmonary Infection Score (CPIS)}

Clinical Pulmonary Infection Score was found to be more appropriate for the study. CPIS score was developed for the diagnosis of Pneumonia (Pugin, 2002). CPIS uses seven criteria for assessment or sometimes five criteria. It combines radiographic, microbiological, physiologic and clinical data into a score. The variables of CPIS score are Temperature, Blood Leukocyte Count, Tracheal secretion, Oxygenation, Pulmonary radiograph. This tool demonstrated a sensitivity of $93 \%$ and a specificity of $100 \%$ in the diagnosis of Pneumonia. The Cohen Kappa value of CPIS is 0.81 , indicating a good validity of the scale.

In the present study, Clinical Pulmonary Infection Score was used for two purposes. At day one CPIS was used to compile a baseline score; to screen and exclude the patient with Pneumonia. On day two or by 48 hours, CPIS was used to screen and exclude patients with Hospital associated Pneumonia. At day six, CPIS was used to check the Progression of Pneumonia and to evaluate the effectiveness of Oral Care. Progression of Pulmonary infiltrates and Culture of the tracheal aspirate. The maximum possible score is 12 . A score of $\geq$ 6 is considered as high likelihood of pneumonia and score $<6$ is considered as low likelihood of pneumonia. Criteria number 6, Progression of the Pulmonary Infiltrates ee and criteria 7 related to, Culture of Tracheal Aspirate ${ }^{\text {ec }}$ may not be feasible to assess at the time of intubation.

\section{Microbiological Profile}

Microbiological Profile includes Culture of Endotracheal secretion, Endotracheal Gram Stain and Acid-Fast Bacilli Stain. Endotracheal culture was done to detect growth of microorganism. Endotracheal gram stain was included to screen Gram Positive and GramNegative microorganism and Acid-Fast Bacilli Stain for the presence of myco- bacterium tuberculin and fungal growth. Endotracheal secretion culture reported no growth or growth of various bacteria and also the bacterial count when growth present. It was classified as, "No Bacterial Growth" when no bacteria were seen, "Insignificant Growth" when the colony count was $<10,000$ cfu (Colony forming Unit) and "Significant Growth" if the colony count $>10,000$ cfu and it was confirmed as case of Pneumonia. Microbiological Profile routinely assessed at the time of intubation, on the third and sixth day after intubation was documented.

\section{Modified Beck Oral Assessment Scale}

This scale includes five components like lips, gingiva, oral mucosa, tongue, teeth and saliva. The total score ranges from 5-20. A score of 5 indicates No Dysfunction and requires a $12^{\text {th }}$ hourly care; a score of 610 indicates Mild Dysfunction and requires an 8th to 12th hourly care; a score of 11-15 indicates Moderate Dysfunction and require an 8th hourly oral care and a score of 16-20 indicates Severe Dysfunction and requires a $4^{\text {th }}$ hourly care.

\section{Data Collection Procedure}

Ethical permission was taken from institution ethical committee. Consent was obtained from the family members as the patient was on ventilator. The data was collected from $1^{\text {st }}$ January 2018 to $1^{\text {st }}$ March 2018. Patient less than 18 years of age and CLD Patients with Endotracheal intubation performed within the past 24 hours were included in the study, whereas ventilated patients with excessive bleeding having oral packing or nasal bleed, diagnosed with pneumonia on admission or by 48 hours of intubation as assessed by a Clinical Pulmonary Infection Score of $\geq 6$ were excluded from the study.

\section{RESULTS}

\section{Results Related to Socio Demographic and Clinical Variables}

Socio Demographic Variables like Age, Gender, History of Smoking, History of Alcohol intake and Tobacco Chewing and the Clinical variables like Diagnosis of patient, Antifungal, Anti- Tubercular and Anti-Viral medications. Chi Square was computed to test the homogeneity of the Study and Comparison group at the baseline. Both the groups were statistically identical in the selected characteristics or drawn from the same population. 
ORAL CARE PROTOCOL OF PNEUMONIA AMONG VENTILATED PATIENTS

Table 1: Frequency and Percentage Distribution of the Socio Demographic and Clinical Variables

$\mathbf{n}_{1}+\mathbf{n}_{2}=27+23$

\begin{tabular}{|c|c|c|c|c|c|c|c|}
\hline \multirow{3}{*}{ Sample characteristics } & \multicolumn{4}{|c|}{ Groups } & \multirow{3}{*}{$\chi^{2}$} & \multirow{3}{*}{$d f$} & \multirow{3}{*}{$p$ value } \\
\hline & \multicolumn{2}{|c|}{ Study (n 1$)$} & \multicolumn{2}{|c|}{ Comparison (n $\left.\mathbf{n}_{2}\right)$} & & & \\
\hline & f & $\%$ & f & $\%$ & & & \\
\hline \multicolumn{8}{|l|}{ Age (in years) } \\
\hline$\leq 40$ & 09 & 33.3 & 03 & 13.0 & \multirow{2}{*}{2.80} & \multirow{2}{*}{1} & \multirow{2}{*}{0.09} \\
\hline$>40$ & 18 & 66.6 & 20 & 86.9 & & & \\
\hline \multicolumn{8}{|l|}{ Gender } \\
\hline Male & 23 & 85.2 & 18 & 78.3 & \multirow{2}{*}{0.40} & \multirow{2}{*}{1} & \multirow{2}{*}{0.53} \\
\hline Female & 04 & 14.8 & 5 & 21.7 & & & \\
\hline \multicolumn{8}{|l|}{ History of smoking } \\
\hline Yes & 10 & 37.0 & 04 & 17.4 & \multirow{2}{*}{2.38} & \multirow{2}{*}{1} & \multirow{2}{*}{0.12} \\
\hline No & 17 & 63.0 & 19 & 82.6 & & & \\
\hline \multicolumn{8}{|l|}{ History of alcohol intake } \\
\hline Yes & 17 & 63.0 & 11 & 47.8 & \multirow{2}{*}{1.16} & \multirow{2}{*}{1} & \multirow{2}{*}{0.28} \\
\hline No & 10 & 37.0 & 12 & 52.2 & & & \\
\hline \multicolumn{8}{|l|}{ History of tobacco use } \\
\hline Yes & 07 & 25.9 & 04 & 17.4 & \multirow{2}{*}{0.53} & \multirow{2}{*}{1} & \multirow{2}{*}{0.47} \\
\hline No & 20 & 74.1 & 19 & 82.6 & & & \\
\hline \multicolumn{8}{|l|}{ Diagnosis } \\
\hline CLD Ethanol & 16 & 59.2 & 17 & 73.9 & \multirow{2}{*}{1.19} & \multirow{2}{*}{1} & \\
\hline CLD & 11 & 40.7 & 06 & 26.0 & & & 0.28 \\
\hline Antifungal & & & & & & & \\
\hline Yes & 21 & 77.8 & 14 & 60.9 & & & \\
\hline No & 06 & 22.2 & 09 & 39.1 & 1.69 & 1 & 0.19 \\
\hline Anti-tubercular drugs & & & & & & & \\
\hline Yes & 0 & 0 & 01 & 4.3 & & & \\
\hline No & 27 & 100 & 22 & 95.7 & 1.19 & 1 & 0.27 \\
\hline Anti-viral drug & & & & & & & \\
\hline Yes & 05 & 18.5 & 06 & 26.1 & & & \\
\hline No & 22 & 81.5 & 17 & 73.9 & 0.41 & 1 & 0.52 \\
\hline
\end{tabular}

$p>0.05 ;$ Not Significant

Comparison of Oral Health Status Between Study and Comparison Group

Table 2: Repeated Measure ANOVA of Oral Health Status of Study and Comparison Group

$\mathrm{n}_{1}+\mathrm{n}_{2}=\mathbf{2 7}+\mathbf{2 3}$

\begin{tabular}{|c|c|c|c|c|c|}
\hline \multirow[t]{2}{*}{ Groups } & \multicolumn{3}{|c|}{$\begin{array}{c}\text { Oral Health Status (MBOAS score) } \\
\text { Mean } \pm \text { SD }\end{array}$} & \multirow[t]{2}{*}{ Time F (p) } & \multirow[t]{2}{*}{ Group $\times$ Time F $(p)$} \\
\hline & Day 1 & Day 3 & Day 6 & & \\
\hline $\begin{array}{l}\text { Study } \\
\left(\mathrm{n}_{1}\right)\end{array}$ & $9.74 \pm 1.58$ & $9.30 \pm 1.54$ & $7.56 \pm 2.62$ & \multirow{2}{*}{$5.82(0.001)^{*}$} & \multirow{2}{*}{$19.16(0.001)^{*}$} \\
\hline$\underset{<<0.01 ; * \text { significant }}{\text { Comparison }\left(\mathrm{n}_{2}\right)}$ & $8.96 \pm 1.77$ & $9.39 \pm 2.08$ & $9.61 \pm 2.35$ & & \\
\hline
\end{tabular}

66| VOL. 13 (2) October 2021 | THE MALAYSIAN JOURNAL OF NURSING 
To determine the effectiveness, a repeated measures ANOVA was computed. Table 2 depict the Mean, Standard Deviation, ANOVA and $p$ value of Oral Health Status of the Study and Comparison group. It can be seen that in the Study group, the mean Oral Health Status score gradually decreased from day one (x
$=9.74 \pm 1.5 \mathrm{SD})$ to Day three $(\mathrm{x}=9.30 \pm 1.54 \mathrm{SD})$ and further decreased to $(\mathrm{x}=7.56 \pm 2.62 \mathrm{SD})$ at day six. In the Comparison group, the mean Oral Health Status score was $(\mathrm{x}=8.96 \pm 1.77 \mathrm{SD})$ at day one which increased to $(\mathrm{x}$ $=9.39 \pm 2.08 \mathrm{SD})$ at day three and further increased to $(\mathrm{x}$ $=9.61 \pm 2.35 \mathrm{SD})$ at day six.

Table 3: Chi square Comparison of Occurrence of Pneumonia in the Study and Comparison group on Day 3 and Day 6

\begin{tabular}{|c|c|c|c|c|c|c|c|}
\hline \multirow{3}{*}{$\begin{array}{l}\text { Culture of } \\
\text { Endotracheal } \\
\text { Secretion }\end{array}$} & \multicolumn{4}{|c|}{ Baseline } & \multirow{3}{*}{$\chi^{2}$} & \multirow{3}{*}{$d f$} & \multirow{3}{*}{$\boldsymbol{P}$} \\
\hline & \multicolumn{2}{|c|}{ Study $\left(n_{1}\right)$} & \multicolumn{2}{|c|}{ Comparison (n2) } & & & \\
\hline & f & $\%$ & $\mathbf{f}$ & $\%$ & & & \\
\hline \multicolumn{8}{|l|}{ Day 3} \\
\hline No Bacterial Growth & 12 & 44.4 & 03 & 13.0 & \multirow{3}{*}{5.83} & \multirow{3}{*}{2} & \multirow{3}{*}{$0.05^{*}$} \\
\hline Insignificant Growth & 12 & 44.4 & 16 & 69.6 & & & \\
\hline Significant Growth (VAP) & 03 & 11.1 & 04 & 17.4 & & & \\
\hline \multicolumn{8}{|l|}{ Day 6} \\
\hline No Bacterial Growth & 12 & 44.4 & 03 & 13.0 & \multirow{3}{*}{6.31} & \multirow{3}{*}{2} & \multirow{3}{*}{$0.04^{*}$} \\
\hline Insignificant Growth & 10 & 37.0 & 11 & 47.8 & & & \\
\hline Significant Growth (VAP) & 05 & 18.5 & 09 & 39.1 & & & \\
\hline
\end{tabular}

Comparison of Occurrence of Pneumonia between Study and Comparison Group

On day three, in the Study group $44.4 \%$ ventilated patients had No Bacterial Growth, 44.4\% had Insignificant Growth and $11.1 \%$ had Significant Growth while in the Comparison group 13\% had No Bacterial Growth, 69.6\% had Insignificant Growth and 17.4 had Significant Growth. On day six, $44.4 \%$ patients were having No Bacterial Growth, $37.0 \%$ having Insignificant Growth, 18.5\% had Significant Growth in the Study group while $13.0 \%$ had No Bacterial Growth, $47.8 \%$ had Insignificant Growth and 39.1\% had Significant Growth in the Comparison group. Significant Growth of microorganism in the ET culture of more than $10,000 \mathrm{cfu}$ was confirmatory for development of Ventilator associated Pneumonia (VAP).

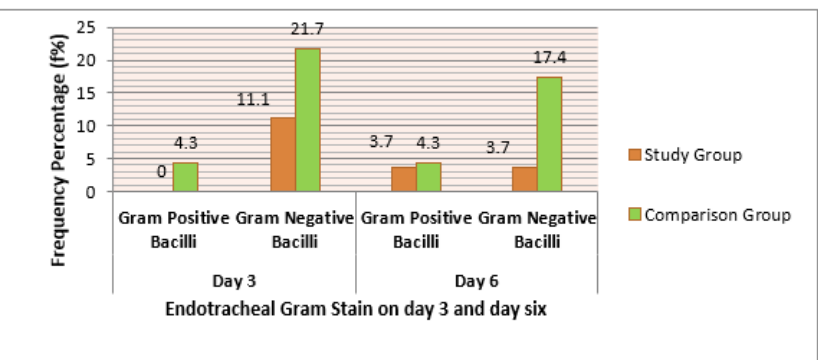

Figure 1: Clustered Column Chart Showing the Frequency Percentage Distribution of Patients Based on Gram Negative and Gram-Positive Bacilli Seen in ET Gram Stain on Day 3 and Day 6

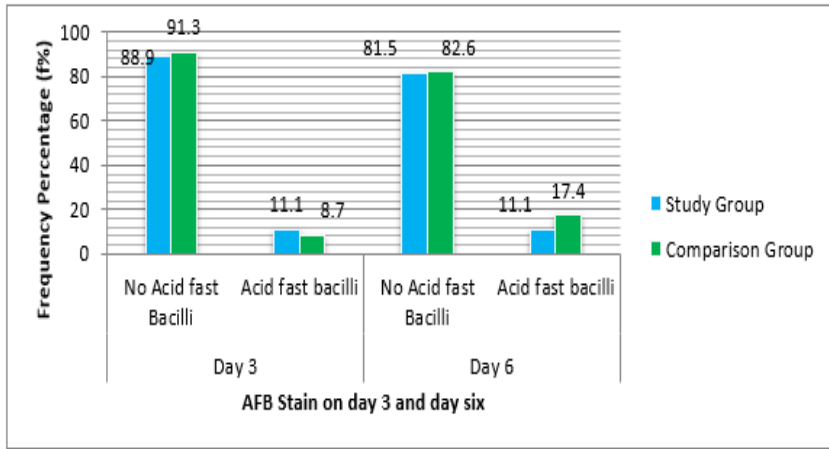

Figure 2: Clustered Column Chart Showing the Frequency Percentage Distribution of Patients Based on Gram Negative and Gram-Positive Bacilli Seen in AFB Stain on Day 3 and Day 6

Comparison of the Progression of Pneumonia in Study and Comparison group

Table 4: Mean Progression of Pneumonia of Study and Comparison group between Day 1 and Day 6

$$
n_{1}+n_{2}=27+23
$$

\begin{tabular}{|c|c|c|}
\hline \multirow{2}{*}{ Groups } & \multicolumn{2}{|c|}{ Progression of Pneumonia by CPIS } \\
\cline { 2 - 3 } & \multicolumn{2}{|c|}{ Mean \pm SD } \\
\cline { 2 - 3 } & Day 1 & Day 6 \\
\hline Study $\left(\mathrm{n}_{1}\right)$ & $3.15 \pm 1.06$ & $4.10 \pm 1.51$ \\
\hline Comparison $\left(\mathrm{n}_{2}\right)$ & $3.22 \pm 1.20$ & $5.58 \pm 1.67$ \\
\hline$p>0.05 ;$ Not Significant & \multicolumn{2}{|}{} \\
\hline
\end{tabular}


Mean CPIS score for the Study group on days one and day six were $(3.15 \pm 1.0$ SD-) and (4.10 \pm 1.51 SD), respectively. In the Comparison group, the mean CPIS score was $(3.22 \pm 1.20 \mathrm{SD})$ and $(5.58 \pm 1.67 \mathrm{SD})$ on day one and day six respectively. One Way ANOVA shows that there was a significant difference from days one to six with time $(F=39.90 ; p=0.00)$. A significant difference was also seen between the groups and time $(F=5.14, p=0.03)$. In addition, the two groups also differed overall $(F=4.34$; $p=0.04$ ) the Comparison group had a higher mean than the Study group.

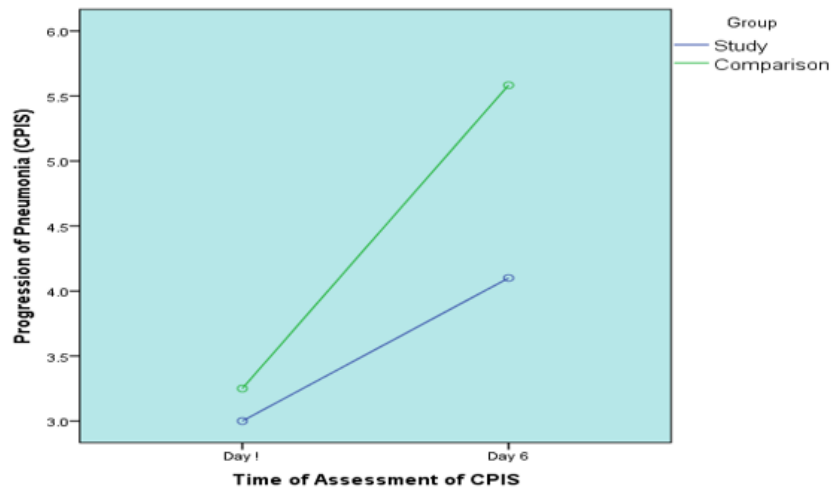

Figure 3. Line Graph Showing the Mean of Progression of Pneumonia (CPIS) of Study and Comparison Group Between Day One and Day Six

\section{Relationship of Severity of Illness with Progression} of Pneumonia

Table 5: Relationship of mean Severity of Illness with mean Change in Oral Health Status in Study and Comparison Group

\begin{tabular}{|l|c|c|c|c|}
\hline Groups & $\begin{array}{c}\text { Mean } \\
\text { Severity } \\
\text { of illness }\end{array}$ & $\begin{array}{c}\text { Mean Difference } \\
\text { of Oral Health } \\
\text { Status Score of } \\
\text { Day 1 to Day 6 }\end{array}$ & $\boldsymbol{r}$ & $\boldsymbol{P}$ \\
\hline $\begin{array}{l}\text { Study } \\
\left(\mathrm{n}_{1}\right)\end{array}$ & 21.63 & -1.89 & 0.16 & 0.41 \\
\hline $\begin{array}{l}\text { Comparison } \\
\left(\mathrm{n}_{2}\right)\end{array}$ & 23.30 & 0.65 & - & 0.83 \\
\hline \multicolumn{2}{|l|}{$>0.05 ;$ Not Significant }
\end{tabular}

To find out the relationship between mean Severity of Illness and mean Progression of Pneumonia, Pearson Correlation was calculated. In the Study group, the correlation on day one $(r=0.16 ; p=0.41)$, and day two $(r=0.16 ; p=0.41)$ was not found statistically significant. The correlation was found statistically significant at 0.05 level of significance on day six $(r=0.66 ; p=0.001)$. In Comparison group, correlation between Severity of Illness and mean Progression of Pneumonia on Day one $(r=0.17 ; p=0.43)$, day two $(r=0.09 ; p=0.67)$ and on day six $(r=0.43 ; p=0.15)$ were not found statistically significant at 0.05 level of significance.

\section{DISCUSSION}

In the present study, the Need Based Oral Care was provided to the patients in the study group. Need was identified in terms of frequency of Oral Care which was determined using MBOAS. The present study findings revealed that all the patients in the study group require 8th hourly oral care as determined by MBOAS, while the patients in the Comparison group received routine oral care as per manual of ILBS (suggest $4^{\text {th }}$ hourly oral care). So, it can be concluded that although there was no complication seen in any of the patients in the Study group, the care provided 8th hourly as per AACN was found to be significant effective as compare to the routine oral care 4 th hourly.

Similarly, the American Association of Critical Care Nurse guidelines recommend assessment of the oral cavity and lips every $8^{\text {th }}$ hourly in ventilated patients (Sarangi, Sarangi, \& Solaman, 2021). After providing oral care every 8th hourly, the incidences of VAP reduced to zero (Munro, \& Grap, 2004).

In reference to Oral Health Status, the data finding showed that there was no significant difference $\left(\chi^{2}=1.64, p=0.20\right)$ found in the Oral Health Status between study and comparison group on the first day whereas the mean MBOAS score decreased significantly $(F=5.82, p=0.001)$ with time and between the groups and time $(F=19.16, p=0.001)$ from day one to day six. Hence the present study found improvement in Oral Health Status of the Study group with the implementation of Need Based Oral Care. The finding of the present study was supported by Ames et al (2011) who conducted a study to assess the effect of systematic oral care in critically ill patients $(n=116)$. The result of the study revealed that MBOAS score differed significantly $(p=0.01)$ across the time. The study concluded that Oral Health Status improved after nurses implemented oral care protocol for oral care. Similar findings reported by Handa et al (2014) and Kumari et al., (2013) who reported that a statistically significant difference $(t=9.17, p=0.05),(t=8.90 ; p=0.001)$ was found in the mean oral health status score of the study group. The study concluded that oral care as per protocol was effective in improving the oral health assessment score of the subjects. 
The findings of the present study were consistent with a number of previous studies showing improvement in Oral Health Status with the Implementation of Oral Care Protocol (Moustafa et al., (2016) \& Duzkaya et al., (2017). In the present study, to assess the effectiveness of Oral Care Protocol, Occurrence of Pneumonia was defined in terms of Microbiological Profile, showing significant growth of Microorganism in the ET culture. Microbiological Profile was done on day 3 and 6 . The reports on both days showed major proportion of patients in both the groups had insignificant, No growth of the microorganism in the ET culture. On day 3, a significant growth was present in $11.1 \%$ of patients among Study group and $17.4 \%$ of the patients in the Comparison group, which directly shows the presence of VAP. On comparison of Occurrence of VAP on day 3 to day 6 , the rate of occurrence of VAP was found to higher in both study (18.5\%) and Comparison (39.1\%). Moreover, there was significant difference found in terms of Occurrence of Pneumonia between the study group and comparison group $\left(\chi^{2}=5.83, p=0.05\right)$ on day 3 and day six $\left(\chi^{2}=6.31, p=0.04\right)$. Hence it can be interpreted that there was significantly higher occurrence of Pneumonia in the Comparison group on both the days. These findings indicated that the oral care protocol was effective in reducing the Occurrence of VAP in ventilated patients.

In the present study, for assessment of Progression of Pneumonia CPIS was used. The results of the study found that during the screening of the patients on day one, there was no significant difference $(t=0.21, p=0.83)$ found between the two groups. The study also found that there is an increase in the mean CPIS of day one to day six for both the groups. However, the increase in the mean CPIS in the Comparison group was significantly higher than the Study group. It was also found that there was a significant difference between the groups from day one to day six with time $(F=39.90, p=0.001)$ and between the groups and time $(F=4.34, p=0.04)$. This may be attributed to that the Need Based Oral Care Protocol was effective in reducing the Progression of Pneumonia.

These findings were consistent with study conducted by Moustafa et al., (2016) who conducted a study to see the effect of Oral Care Intervention on the occurrence of Ventilator-associated Pneumonia among 60 ventilated patients at Egypt. Clinical Pulmonary Infection score was used to assess the patient for Pneumonia. The results of the study revealed that there was a statically significant difference $(p=0.001)$ found in the occurrence of VAP after implementation of Oral Care Protocol.

The findings of the present study were also supported by Orr and Mitchell (2018) who undertook a study to evaluate the effectiveness of comprehensive oral care hygiene protocol in prevention of pneumonia at Nebraska Methodist Hospital, Omaha, Nebraska. Results of study revealed that Pneumonia rates decreases after implementation of Oral Care Protocol.

However, there is a study done by Haghighi et al., (2017), which shows inconsistent result to the present study. The results of the study reported that there was no statistically significant decrease in the incidence of ventilator-associated pneumonia in critically ill patients compared to the routine oral care practices.

Hence the present study found that Need Base Oral Care Protocol was effective in improving the Oral Health Status, Occurrence and Progression of Pneumonia among Ventilated patients which is supported by number of existing studies.

To identify the relationship of Severity of Illness with change in Oral Health Status and progression of pneumonia Pearson correlation was calculated. Change in Oral Health Status refers to the difference in the mean MBOAS score from day one to six. Progression of Pneumonia was depicted in terms of the mean CPIS at day one, two and six.

The results of the present study showed that no significant relationship exist between mean Severity of Illness and Change in Oral Health Status (from day 1 to day 6) in the Study $(r=0.16, p=0.41)$ and Comparison $(r=-0.04, p=0.83)$ group.

This revealed that oral Health Status did not change over time in relation to the Severity of Illness. This particular association between Severity of Illness and Change in Oral Health Status has not been studied previously. The present study finding revealed a positive relationship between Severity of illness and Progression of Pneumonia for both the groups at day one, two and six as interpreted from the $r$ value $\left[\mathrm{D}_{1}(r=0.16 \mathrm{vs} 0.17)\right.$, $\left.\mathrm{D}_{2}(\mathrm{r}=0.16 \mathrm{vs} 0.09), \mathrm{D}_{6}(r=0.66 \mathrm{vs} 0.43)\right]$. Furthermore, it was found that a moderate positive significant $(r=0.667$; $p=0.001$ ) relationship seen in the Study group patients at day six.

The findings of the study were supported by Munro et al., (2006) who also found a significant correlation $(p=0.00)$ between Severity of Illness and CPIS at day 4 
among Ventilated Patients. The study concluded that the patient with high severity of illness is at more risk of ventilator associated pneumonia which is consistent with the result of the present study.

\section{CONCLUSION}

The patient with chronic liver disease often require ventilator support due to disease process. Hence the patient required oral care to prevent the occurrence of pneumonia. So, need based oral care protocol was developed. The results of the study showed that the need base oral care was effective in improving the oral health status of the patient.

\section{Conflict of Interests}

The authors declare that there is no conflict of interests.

\section{ACKNOWLEDGEMENT}

The authors are thankful to the of Guide, Co guide, Clinical guide, Colleagues, teacher, parents and many other people for helping them in completing the task within the time.

\section{REFERENCES}

Ames, N. J., Sulima, P., Yates, J. M., McCullagh, L., Gollins, S. L., Soeken, K., \& Wallen, G. R. (2011). Effects of systematic oral care in critically ill patients: a multicenter study. American Journal of Critical Care, 20(5), 103-114.

Antonelli, M., Azoulay, E., Bonten, M., Chastre, J., Citerio, G., Conti, G., De Backer, D., Gerlach, H., Hedenstierna, G., Joannidis, M., Macrae, D., Mancebo, J., Maggiore, S. M., Mebazaa, A., Preiser, J. C., Pugin, J., Wernerman, J., \& Zhang, H. (2011). Year in review in Intensive Care Medicine 2010: III. ARDS and ALI, mechanical ventilation, noninvasive ventilation, weaning, endotracheal intubation, lung ultrasound and paediatrics. Intensive Care Medicine, 37(3), 394-410.

Davis, K. A., (2006). Ventilator-associated pneumonia: a review. Journal of Intensive Care Medicine, 21(4), 211-226.

Diez, M. D. J., Andres, D. L. A., Barrera, H. V., Trujillo, J. I., Bailón, M. M., Yanes, M. D. J., \& Garcia, J. R. (2017). Decreasing incidence and mortality among hospitalized patients suffering a ventilator-associated pneumonia. Medicine, 96(30),7625.

Duzkaya, S. D., Uysal, G., Bozkurt, G., \& Tulay, Y. (2017). The Effect of Oral Care Using an Oral Health Care Guide on Preventing Mucositis in Pediatric Intensive Care. Journal of Pediatric Nursing, 36, 98-102.

Haghighi, A., Shafipour, V., Bagheri-Nesami, M., Baradari, A. G., \& Charati, J. Y. (2017). The impact of oral care on oral health status and prevention of ventilator-associated pneumonia in critically ill patients. Australian Critical Care, 30(2), 69-73.

Handa, S., Chand, S., Sarin, J., Singh, V., \& Sharma, S. (2014). Effectiveness of oral care protocol on oral health status of hospitalised children admitted in intensive care units of selected hospital of Haryana. Nursing and Midwifery Research Journal, 10(1), 8-15.

Joseph, N. M., Sistla, S., Dutta, T. K., Badhe, A. S., \& Parija, S. C. (2009). Ventilator-associated pneumonia in a tertiary care hospital in India: incidence and risk factors. The Journal of Infection in Developing Countries, 3(10), 771-777.

Kannan, N., Prathima, P., Rakesh Kumar Manne, A. A., Nellore Bhargavi, S., Pulimi, A. N., Sravanthi, M., \& Havilah, P. (2018). Oral Manifestations in Patients with Liver Diseases A Prospective Observational Study at A Tertiary Health Care Center in India.

Knaus, W. A., Draper, E. A., Wagner, D. P., \& Zimmerman, J. E. (1985). APACHE II: a severity of disease classification system. Critical Care Medicine, 13(10), 818-829.

Koenig, S. M., \& Truwit, J. D. (2006). Ventilator-associated pneumonia: diagnosis, treatment, and prevention. Clinical Microbiology Reviews, 19(4), 637-657. 
Kumari, N., Kumari, V., Varsha, A. S. V., \& Chandna, S. (2013). Effectiveness Of0.2\% Chlorhexidine and Oral Routine Care in Terms of Oral Health Status and Oral Microbiological Colony Count of Self Care Deficit Clients, Experimental Study. Journal of Dental and Medical Sciences, 11(5), 1-6.

Marshall, J. C., Bosco, L., Adhikari, N. K., Connolly, B., Diaz, J. V., Dorman, T., ... \& Zimmerman, J. (2017). What is an intensive care unit? A report of the task force of the World Federation of Societies of Intensive and Critical Care Medicine. Journal of Critical Care, 37, 270-276.

Moustafa, M. F., Tantawey, N. M., El-Soussi, A. H., \& Ramadan, F.A. (2016). The effect of oral care intervention on the occurrence of ventilator-associated pneumonia. Gynecol Obstet (Sunnyvale), 6(383), 2161-0932.

Munro, C. L., \& Grap, M. J. (2004). Oral health and care in the intensive care unit: state of the science. American Journal of Critical Care, 13(1), 25-34.

Munro, C. L., Grap, M. J., Elswick Jr, R. K., McKinney, J., Sessler, C. N., \& Hummel III, R. S. (2006). Oral health status and development of ventilator-associated pneumonia: a descriptive study. American Journal of Critical Care, 15(5), 453-460.

Orr, J., \& Mitchell, M. (2008). Prevention of hospital-associated pneumonia using a comprehensive oral hygiene protocol.

Panov, V., \& Krasteva, A. (2011). Oral health in patients with liver diseases. JIMAB, 17, 140-142.

Pugin, J. (2002). Clinical signs and scores for the diagnosis of ventilator-associated pneumonia. Minerva Anestesiologica, 68(4), 261-265.

Sarangi, A., Sarangi, S., \& Solaman, L. (2021). Oral care strategies in patients in intensive care units. The Southwest Respiratory and Critical Care Chronicles, 9(39), 48-52. 